\title{
Regulatory $T$ cells participate in the recovery of ischemic stroke patients
}

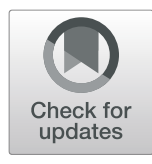

\author{
María Santamaría-Cadavid, Emilio Rodríguez-Castro, Manuel Rodríguez-Yáñez, Susana Arias-Rivas, \\ Iria López-Dequidt, María Pérez-Mato, Manuel Rodríguez-Pérez, Ignacio López-Loureiro, Pablo Hervella, \\ Francisco Campos, José Castillo, Ramón Iglesias-Rey ${ }^{*}$ (D) and Tomás Sobrino*
}

\begin{abstract}
Background: Recent preclinical studies have shown that regulatory T cells (Treg) play a key role in the immune response after ischemic stroke (IS). However, the role of Treg in human acute IS has been poorly investigated. Our aim was to study the relationship between circulating Treg and outcome in human IS patients.

Methods: A total of 204 IS patients and 22 control subjects were recruited. The main study variable was good functional outcome at 3 months (modified Rankin scale $\leq 2$ ) considering infarct volume, Early Neurological Deterioration (END) and risk of infections as secondary variables. The percentage of circulating Treg was measured at admission, 48, $72 \mathrm{~h}$ and at day 7 after stroke onset.

Results: Circulating Treg levels were higher in IS patients compared to control subjects. Treg at $48 \mathrm{~h}$ were independently associated with good functional outcome (OR, 3.5; Cl: 1.9-7.8) after adjusting by confounding factors. Patients with lower Treg at $48 \mathrm{~h}$ showed higher frequency of END and risk of infections. In addition, a negative correlation was found between circulating Treg at $48 \mathrm{~h}(r=-0.414)$ and $72 \mathrm{~h}(r=-0.418)$ and infarct volume.
\end{abstract}

Conclusions: These findings suggest that Treg may participate in the recovery of IS patients. Therefore, Treg may be considered a potential therapeutic target in acute ischemic stroke.

Keywords: Early neurological deterioration, Interleukin-10, Ischemic stroke, Neuroinflammation, Regulatory T cells, Risk factors

\section{Background}

Stroke represents the second cause of death in Europe and developed countries. In addition to mortality, longterm morbidity remains as one of the main consequence associated to stroke patients [1, 2]. Although stroke causes enormous medical and economic costs associated to stroke, thrombolysis with recombinant plasminogen activator (rtPA) remains as the only approved pharmacological treatment for ischemic stroke (IS). However, there is a narrow therapeutic window for the use of rtPA treatment $(<4.5 \mathrm{~h})$ due to side effects such as hemorrhagic transformation or treatment with previous

\footnotetext{
* Correspondence: ramon.iglesias.rey@sergas.es;

tomas.sobrino.moreiras@sergas.es

Clinical Neurosciences Research Laboratory, Clinical University Hospital,

Health Research Institute of Santiago de Compostela (IDIS), Hospital Clínico,

c/ Travesa da Choupana, s/n, 15706 Santiago de Compostela, Spain
}

anticoagulants [3]. Because of these limitations, rtPA is only available for a small percentage of IS patients in developed countries, being this situation worst in developing countries. Therefore, new and effective therapies are highly demanded in clinical practice.

Stroke triggers an acute immunological and inflammatory response in the brain that participates actively in the evolution of ischemic damage. In the last years, the interest in the role of inflammation in stroke pathogenesis has significantly increased, becoming an important target for future therapeutic drugs. In this regard, it is well known that decrease of blood flow in a brain area causes neuronal necrosis and leads to an immune response and invasion of inflammatory cells in the ischemic tissue, which mediates a secondary brain injury [46]. However, although immune response contributes to brain tissue damage, therapeutic strategies based on

(c) The Author(s). 2020 Open Access This article is distributed under the terms of the Creative Commons Attribution 4.0 International License (http://creativecommons.org/licenses/by/4.0/), which permits unrestricted use, distribution, and 
immunosuppression have failed to show efficacy in clinical trials [7].

Alternatively to the use of immunosuppressant drugs, other mechanisms involved in the control and regulation of inflammatory response have been recently proposed aimed to prevent brain damage after stroke. In this regard, regulatory $\mathrm{T}$ cells (Treg) are a subgroup of CD4 $\mathrm{T}$ lymphocytes that play an important role in maintaining immune homeostasis, preventing autoimmunity and inflammation. Due to their immunomodulatory function, it has been proposed that Treg may play an important role in the pathophysiology of IS [8].

Several preclinical studies have tested the therapeutic role of Treg in cerebral ischemia, finding that their depletion causes larger infarct volumes [9], while their exogenous administration mediates a protective effect [10]. However, few clinical studies have investigated the role of Treg in IS patients [11-16]. These studies showed controversial results, probably due to the small and heterogenous group of patients included in the studies. Moreover, those clinical trials did not evaluate the relationship between circulating Treg levels with infarct volume, patient's outcome or early neurological deterioration (END). In addition, there is a lack of studies investigating the temporal profile of Treg during acute phase of IS. Similarly, there is no data about the association of Treg and interleukin-10 (IL-10), a cytokine with anti-inflammatory properties, which is considered as the main effector mechanisms of Treg [17]. These data could help to establish the potential use of Treg as a therapeutic target able to improve functional outcome in IS patients.

In this clinical study, we have analyzed the association between circulating levels of Treg with the functional outcome in IS patients. Likewise, it was analyzed if higher levels of Treg are related with smaller infarct volumes and less frequency of END. Finally, we have studied the correlation between circulating Treg and serum levels of IL-10.

\section{Methods}

\section{Patient's characteristics}

Ischemic stroke patients within $12 \mathrm{~h}$ from symptoms onset were prospectively included in the study between April 2013-July 2014. A cohort of control subjects matched by sex and age was included.

Inclusion criteria were: hospitalized patients with firstepisode of IS within $12 \mathrm{~h}$ from symptoms onset; age $>18$ years; previously independent for their daily living activities (modified Rankin Scale $(\mathrm{mRS}) \leq 1$ ).

Exclusion criteria were: presence of intracerebral hemorrhage confirmed by neuroimaging; previous IS; cancer or severe systemic disease that determine a life expectancy lower than 6 months; infections during the last 30 days before admission; chronic inflammatory disease; pregnancy; renal replacement therapy; treatment with steroids, immunosuppressive and immunomodulatory drugs or antibiotics during the last 30 days before admission; periodontal disease; and fever in the previous $72 \mathrm{~h}$ (axillary temperature over $38^{\circ} \mathrm{C}$ ). Patients with active infection (axillary temperature $>37.5^{\circ} \mathrm{C}$ and leukocyte levels $>15,000 / \mu \mathrm{L}$ or $<4000 / \mu \mathrm{L}$ ), cough and spitting, voiding dysfunction, diarrhea and clinical signs of endocarditis or meningitis.

On the other hand, a cohort of subjects without any neurological, inflammatory or infectious disease was included as control group. The selection of these control subjects was made by inviting the patient's relatives to participate in the study. Control subjects were matched to patients by gender and age.

\section{Clinical variables and neuroimaging studies}

All patients were admitted in the Stroke Unit of University Clinical Hospital of Santiago de Compostela and treated according to the guidelines of the Cerebrovascular Diseases Study Group of the Spanish Society of Neurology [18]. Medical history recording demographic data, potential vascular risk factors, blood counts, biochemistry and coagulation tests, 12-lead ECG, chest radiography, carotid and transcranial ultrasonography and Computed Tomography (CT) or Magnetic Resonance Imaging (MRI) were performed at admission.

To evaluate neurologic deficit, the National Institute of Health Stroke Scale (NIHSS) was performed at admission, 24,48 and $72 \mathrm{~h}$, at discharge, and at 3 months. END was defined as an increase of 4 points or more in NIHSS assessment between baseline and any other NIHSS evaluation during the first $72 \mathrm{~h}$. Functional outcome was evaluated at discharge and at 3 months by mRS. NIHSS and mRS were evaluated by internationally certified neurologists. Stroke etiology was classified according to TOAST criteria [19].

We evaluated the incidence of any infection during the hospitalization period. A protocol has been implemented in order to evaluate the presence of infections during the acute phase of stroke. The following tests were performed in those patients who showed an axillar temperature $>37.5^{\circ} \mathrm{C}$ in two different determinations separated by $1 \mathrm{~h}$, or in patients with one axillar temperature determination $>38^{\circ} \mathrm{C}$ : blood counts, biochemistry analysis and blood culture; physicians made a clinical suspicion regarding the infection origin. During the etiological examination of the infection origin, empiric antibiotherapy was started according to clinical suspicion. Once the antibiogram was obtained, specific antibiotic treatment was started in case of positive cultures. 
To evaluate infarct volume, a control CT was performed between 4 th-7th days after IS. Infarct volume was quantified in cubic centimeters $\left(\mathrm{cm}^{3}\right)$ and was assessed according to the formula $0.5 \times \mathrm{AxBxC}$, where $\mathrm{A}$ and $\mathrm{B}$ correspond to higher diameters in perpendicular direction and $\mathrm{C}$ to the number of $10 \mathrm{~mm}$ slices where infarct volume is present [20]. All neuroimaging evaluations were made by the same neuroradiologist blinded to clinical and laboratory data.

\section{Quantification of Treg}

Circulating levels of Treg were measured by flow cytometry according to methods and using the markers described elsewhere [21-23]. Prior to patient's inclusion, we selected 20 IS patients who matched inclusion/exclusion criteria to evaluate the optimal temporal profile for the quantification of Treg during the acute phase of IS. Blood samples were collected with an evacuated tube system (Vacutainer) in EDTA tubes at baseline, 24, 48, $72 \mathrm{~h}$ and at days 4, 5 and 7. Based on to this temporal profile, we obtained blood samples in the more relevant time-points for Treg evaluation (i.e. at admission, 48 and $72 \mathrm{~h}$ and 7 th day).

Blood samples were processed within $3 \mathrm{~h}$ after collection by a single researcher who was blinded to patients' clinical, biochemical or radiological results. Circulating Treg were analyzed for the expression of specific surface antigens with direct flow cytometry (BD FACSAria IIu, BD, Franklin Lakes, NJ, USA). In brief, $50 \mu \mathrm{L}$ of peripheral blood were labelled with $10 \mu \mathrm{L}$ of FITC-conjugated anti-CD4 (BD, Franklin Lakes, NJ, USA), $10 \mu \mathrm{L}$ of PEconjugated anti-CD25 (BD, Franklin Lakes, NJ, USA), and $10 \mu \mathrm{L}$ of Alexa Fluor 647 -conjugated anti-CD127 (BD, Franklin Lakes, NJ, USA) monoclonal antibodies. We considered Treg as CD4+/CD25+/CD127- staining cells in the lymphocyte gate. In all analyses, $2.5 \times 10^{5}$ events were acquired, using a FACSAria IIu analyzer (BD, Franklin Lakes, NJ, USA), and processed using the PC FACSDiva software program (BD, Franklin Lakes, NJ, USA). Treg count was expressed as percentage of Treg over total analyzed lymphocytes.

\section{IL-10 determination}

Blood samples, drawn from all patients at admission, and at $24 \pm 6,48 \pm 12$, and $72 \pm 12 \mathrm{~h}$, were collected in glass chemistry test tubes, centrifuged at $3000 \mathrm{rpm}$ during $10 \mathrm{~min}$, and immediately frozen and stored at $80^{\circ} \mathrm{C}$. Serum levels of IL-10 were measured using an immunodiagnostic IMMULITE 1000 System (Siemens Healthcare España, Madrid, Spain). Determinations were performed in an independent laboratory blinded to clinical and neuroimaging data.

\section{Outcome variables}

The primary endpoint was good functional outcome $(\mathrm{mRS} \leq 2)$ at 3 months. Infarct volume and presence of END were evaluated as secondary outcome variables. The development of infections during hospitalization was recorded as safety variable. Finally, we analyzed the correlation between circulating Treg and serum levels of IL-10 in order to investigate the possible mechanism of action of Treg.

\section{Statistical analysis}

Sample size was calculated using the statistical EPIDAT 3.1 software, considering that those patients within the highest quartile regarding Treg levels during the first week after stroke achieve a $25 \%$ more frequency of good outcome at 3 months compared with those with Treg levels in the lowest quartile. The minimum calculated sample size was 172 patients in order to obtain a statistical power of $80 \%$ with a significant difference level of 0.05 .

Results were expressed as percentages for categorical variables and as mean (SD) or median and range (25th and 75th percentiles) for the continuous variables depending on whether their distribution was normal or not. The Kolmogorov-Smirnov test was used for testing the normality of the distribution. Proportions were compared using the chi-square or Fisher test, while the continuous variables between groups were compared with the Student's t or the Mann-Whitney tests depending on whether their distribution was normal or not, respectively. In case of more than 3 groups, variables were compared using ANOVA test. Bivariate correlations were performed using Pearson's (normally distributed variables) or Spearman (variables without normal distribution) coefficients.

ROC curves were used to establish the best cut-off point for Treg levels that optimally predicted good functional outcome.

The independent association of circulating Treg levels with good functional outcome at 3 months and the risk of infections was assessed by logistic regression analysis; while their independent influence on infarct volume was assessed by multiple linear regression models. Each logistic regression analysis or multivariable linear regression model was adjusted for those significant variables in the bivariate analysis. Residual plots were examined to detect potential non-linear relationships between the outcome variable and continuous independent variables. Results were expressed as adjusted odds ratios (ORs) or Beta estimate with the corresponding 95\% confidence intervals $(95 \% \mathrm{CI})$. A $p$-value $<0.05$ was considered to be statistically significant in all tests. The statistical analysis was conducted in SPSS 20.0 (IBM, Chicago, IL, USA) for Mac. 


\section{Results}

Twenty-two control subjects were included. No differences were found between controls and IS patients regarding age, sex, previous history of hypertension, diabetes, dyslipidemia, coronary disease, peripheral artery disease, and alcohol or tobacco consumption. IS patients showed more prevalence of atrial fibrillation than control subjects ( 42.2 vs. $0 \%, p<0.0001)$.

On the other hand, 335 IS patients admitted in the Stroke Unit within the first $12 \mathrm{~h}$ from stroke onset were evaluated to be included in the study. Among those, 204 patients fulfilled all inclusion criteria and did not fulfill any exclusion criteria. One hundred three patients (50.5\%) were males. Mean age was $71.7 \pm 10.6$ years. The NIHSS score at admission was $8[4,12]$ and the mean infarct volume was $50.8 \pm 88.4 \mathrm{~cm}^{3}$. Regarding stroke etiology, we found 93-cardioembolic (45.6\%), 26-atherothrombotic (12.7\%), 7-lacunar (3.4\%) and 78-undetermined (38.2\%).

Circulating levels of Treg $(0.0222 \pm 0.0177$ vs. $0.0013 \pm$ $0.0009 \% ; p<0.0001)$ as well as IL-10 serum levels $(6.9 \pm$ 1.7 vs. $1.8 \pm 0.1 \mathrm{pg} / \mathrm{mL} ; p<0.0001)$ at admission were higher in IS patients than in control subjects.

The temporal pattern of Treg and IL-10 levels are shown in Fig. 1. We found that circulating Treg were significantly higher at $48,72 \mathrm{~h}$ and day 7 in relation to the baseline measurement. Based on these results, we evaluated this temporal profile in the complete cohort of IS patients included in the study.

\section{Primary endpoint: influence of Treg on functional outcome}

Patients with lower mRS scores at 3 months showed higher levels of Treg at 48, $72 \mathrm{~h}$ as well as at day 7, but not at admission (Fig. 2). Table 1 shows the baseline clinical characteristics, vascular risk factors, stroke subtype, biochemical/cellular parameters and Treg levels of patients with good $(n=87 ; 36.2 \%)$ and poor outcome $(n=117 ; 63.8 \%)$ at 3 months. We found that patients with good outcome had higher levels of Treg at $48 \mathrm{~h}$ $(p<0.0001), 72 \mathrm{~h}(p<0.0001)$ and 7 days $(p=0.001)$, but not at admission $(p=0.962)$.

ROC analysis showed that Treg levels at $48 \mathrm{~h} \geq$ $0.0550 \%$ suggested good functional outcome 3 months with a specificity of $97 \%$ and a sensitivity of $95 \%$ (area under the curve: $0.990 ; 95 \%$ CI: $0.997-1.000 ; p<0.0001$ ). Similarly, Treg levels at $72 \mathrm{~h} \geq 0.0650 \%$ suggested good outcome at 3 months with a specificity of $95 \%$ and a sensitivity of $93 \%$ (area under the curve: $0.964 ; 95 \%$ CI: $0.886-1.000 ; p<0.0001)$.

In the logistic regression analysis, Treg levels at $48 \mathrm{~h}$ were independently associated with good functional outcome at 3 months (OR 3.5; 95\% CI: 1.9-7.8; $p<0.0001$ ) after adjustment by age, previous history of hypertension, dyslipemia, atrial fibrillation, leukocyte counts, glucose and fibrinogen levels, high- sensitive C-reactive protein levels, basal NIHSS and cardioembolic stroke. Treg levels at $72 \mathrm{~h}$ were independently associated with good functional outcome at 3 months (OR 1.7; 95\% CI: $1.1-3.1 ; \quad p=0.016)$ after adjustment by the same variables.

\section{Early neurological deterioration (END)}

END was observed in 13 patients (6.4\%). Circulating Treg levels at $48 \mathrm{~h}(0.0132 \pm 0.0125 \%$ vs. $0.0411 \pm$ $0.026 \% ; \quad p<0.0001)$ and $72 \mathrm{~h} \quad(0.0096 \pm 0.0061 \%$ vs. $0.0453 \pm 0.0234 \% ; p<0.0001)$ were lower in patients who
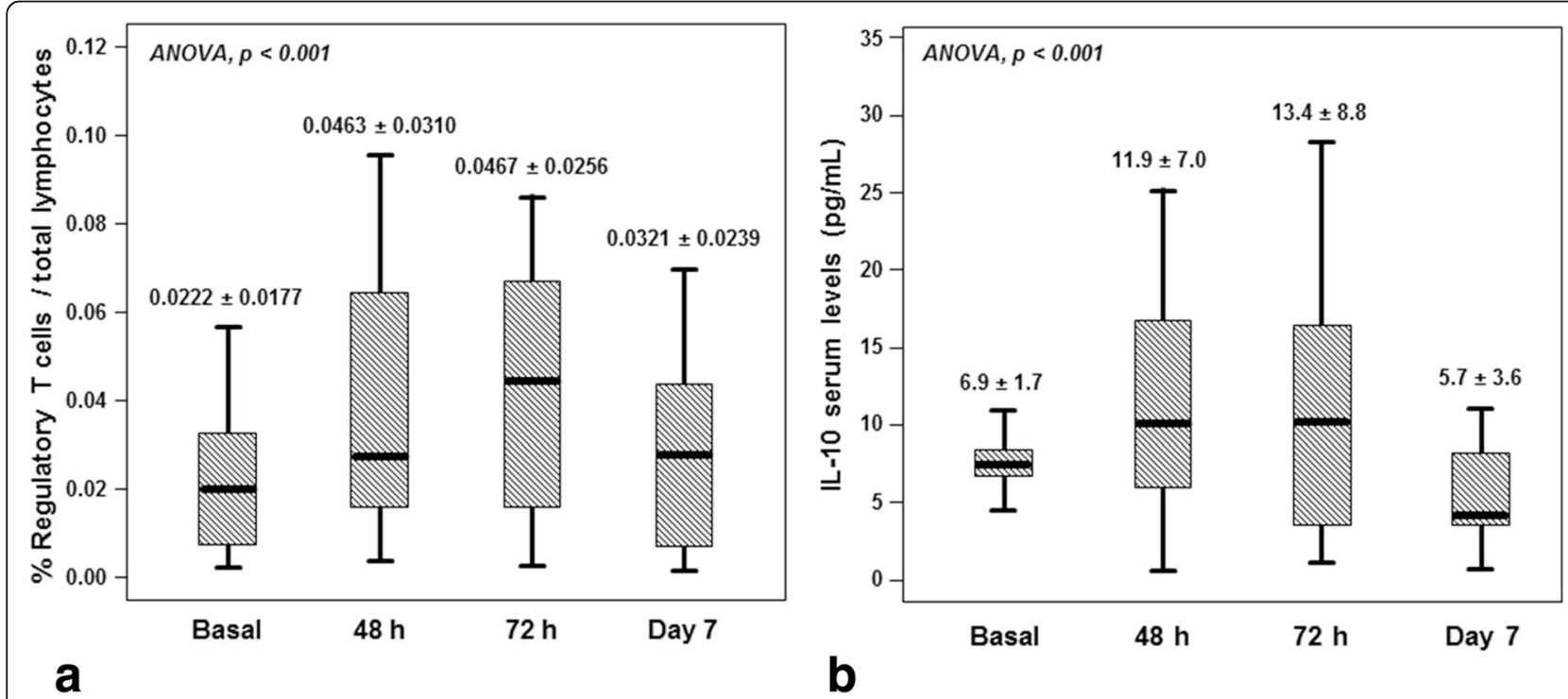

a

Fig. 1 Temporal pattern of \% Treg (a) and IL-10 levels (pg/mL) (b) at admission, 48 and $72 \mathrm{~h}$ and day 7 

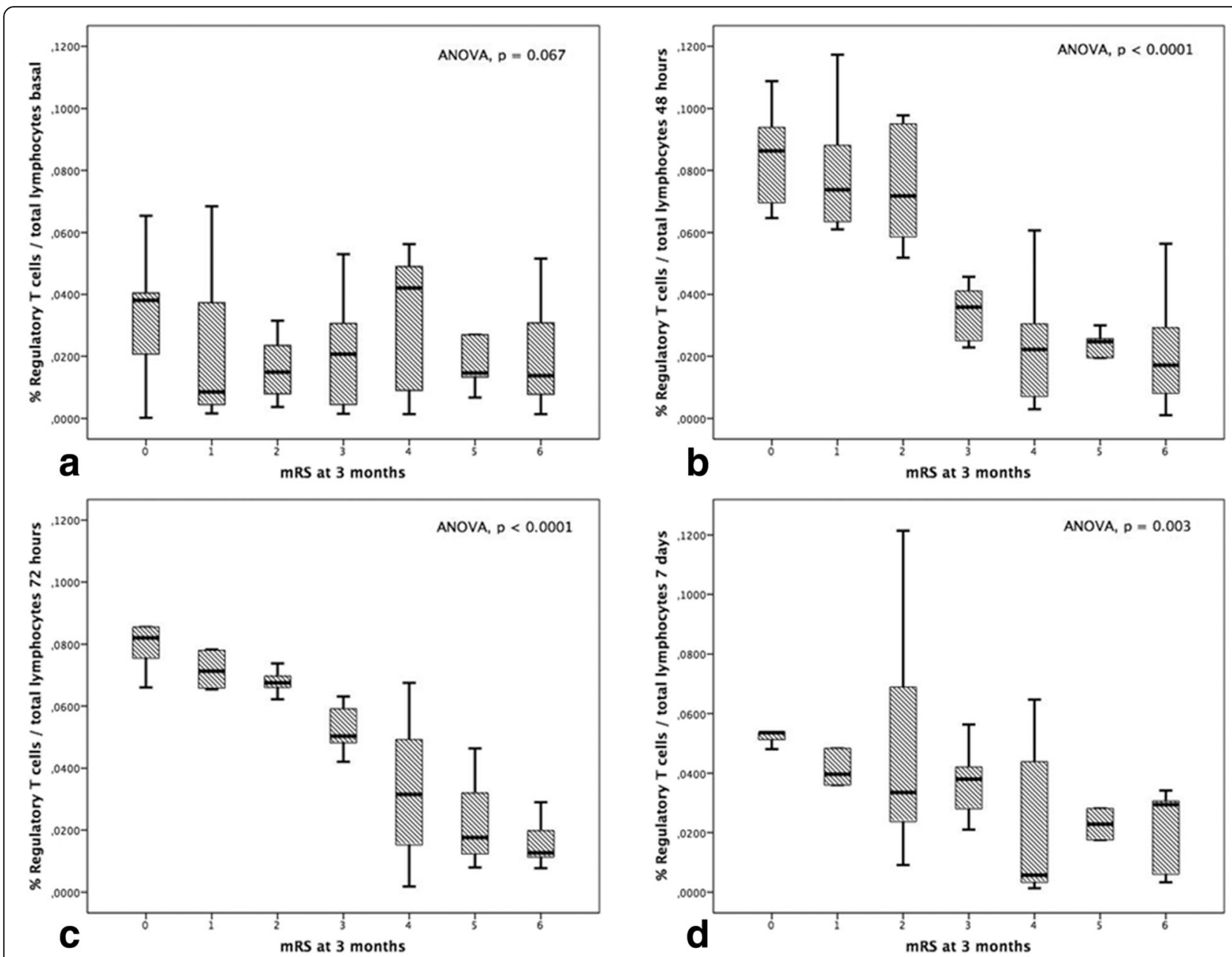

Fig. 2 Association between mRS score at 3 months and circulating levels of Treg at admission (a), $48 \mathrm{~h}(\mathbf{b}), 72 \mathrm{~h}$ (c) and day 7 (d)

suffered END, however, due to the small number of patients with END it was not possible to perform a logistic regression analysis.

\section{Infarct volume}

Infarct volume was measured in 195 patients. We found a negative correlation between infarct volume and circulating levels of Treg at $48 \mathrm{~h}(r=-0.414 ; p<0.0001)$ and $72 \mathrm{~h}(r=-0.418 ; p<0.0001)$. No correlation has been found between infarct volume and Treg levels at baseline and at day 7 .

In the multivariate analysis, Treg levels at $72 \mathrm{~h}$ (B: -648.9 ; 95\% CI: -1251.2 to $-46.8 ; p=0.035)$, but not at 48 h (B: $-545.4 ; 95 \%$ CI: -1036.5 to $291.6 ; p=0.199$ ) were independently associated with the infarct volume after adjustment by age, previous history of hypertension, dyslipemia, atrial fibrillation, leukocyte counts, glucose and fibrinogen levels, high-sensitive C-reactive protein levels, basal NIHSS and cardioembolic stroke.

\section{Risk of infections}

Twenty-six patients (12.7\%) developed infections during the hospitalization period: 17 (65.4\%) had respiratory infections, $6(23.1 \%)$ urinary infections and in 3 patients (11.5\%) the origin was unknown. Infection during hospitalization was associated with higher temperature at $24 \mathrm{~h}\left(38.1 \pm 0.3^{\circ} \mathrm{C}\right.$ vs. $\left.36.6 \pm 0.5^{\circ} \mathrm{C} ; p<0.0001\right)$ and at $48 \mathrm{~h}\left(37.9 \pm 0.5^{\circ} \mathrm{C}\right.$ vs. $\left.36.7 \pm 0.5^{\circ} \mathrm{C} ; p<0.0001\right)$, and with a greater neurological deficit at admission (NIHSS 14[11, $20]$ vs. $9[5,18] ; p=0.033)$. The presence of infections during the hospitalization was associated with poor functional outcome at 3 months; patients with infection showed higher scores of mRS at 3 months $(5[4,6]$ vs. 3 $[1,4] ; p<0.0001)$.

Circulating Treg levels at $48 \mathrm{~h}$ were lower in patients with infections $(0.0189 \pm 0.009 \%$ vs. $0.0425 \pm 0.0280 \%$; $p<0.0001)$. Similar results were found for Treg levels at 72 h $(0.0168 \pm 0.0105 \%$ vs. $0.0473 \pm 0.0238 \%$; $p<0.0001)$.

In the logistic regression analysis, lower Treg levels at $48 \mathrm{~h}$ (OR: $0.35 ; 95 \%$ CI: $0.00-0.57 ; p=0.001$ ) and $72 \mathrm{~h}$ 
Table 1 Baseline clinical characteristics, vascular risk factors, stroke subtype, biochemical/cellular parameters and neuroimaging findings in patients with good or poor outcome at 3 months

\begin{tabular}{|c|c|c|c|}
\hline Variable & $\begin{array}{l}\text { Good outcome } \\
n=87\end{array}$ & $\begin{array}{l}\text { Poor outcome } \\
n=117\end{array}$ & $P$ value \\
\hline Age (years) & $57.4 \pm 8.4$ & $76.3 \pm 7.3$ & $<0.0001$ \\
\hline Female gender, $\mathrm{n}(\%)$ & $36(42.7)$ & $67(57.3)$ & 0.548 \\
\hline Previous hypertension, n (\%) & $32(36.9)$ & $74(63.2)$ & 0.030 \\
\hline Previous diabetes, n (\%) & $31(36.2)$ & $75(64.1)$ & 0.197 \\
\hline Previous dyslipidemia, n (\%) & 28(32.5) & $79(67.5)$ & 0.016 \\
\hline Previous atrial fibrillation, n (\%) & $40(46.7)$ & 63(53.8) & $<0.0001$ \\
\hline Previous ischemic cardiopathy, n (\%) & $65(75.0)$ & $29(24.7)$ & 0.059 \\
\hline Previous peripheric arteriopathy, n (\%) & $0(0)$ & $100(100)$ & 0.220 \\
\hline Alcohol consumption, n (\%) & $39(45.5)$ & $64(54.7)$ & 0.475 \\
\hline Smoking, n (\%) & $47(54.5)$ & $53(45.3)$ & 0.052 \\
\hline Previous statin consumption, n (\%) & 33(37.9) & $73(62.4)$ & 0.184 \\
\hline Leukocyte at admission $\left(\times 10^{3} / \mathrm{mmc}\right)$ & $8.1 \pm 1.7$ & $8.7 \pm 3.8$ & $<0.0001$ \\
\hline Glucose at admission (mg/dL) & $132.5 \pm 39.3$ & $155.8 \pm 89.2$ & $<0.0001$ \\
\hline Fibrinogen at admission (mg/dL) & $380.0 \pm 64.9$ & $400.4 \pm 101.8$ & $<0.0001$ \\
\hline $\mathrm{CRP}(\mathrm{C}$ reactive protein) (mg/L) & $1.5 \pm 1.7$ & $3.2 \pm 4.8$ & $<0.0001$ \\
\hline Recanalization therapy, n (\%) & 55.6 & 44.4 & 0.433 \\
\hline Basal NIHSS & $4[2,7]$ & $13[7,18]$ & $<0.0001$ \\
\hline TOAST & & & 0.003 \\
\hline - Cardioembolic, n (\%) & $27(31.0)$ & $66(56.4)$ & \\
\hline - Aterothrombotic, n (\%) & $13(14.9)$ & $13(11.1)$ & \\
\hline - Lacunar, n (\%) & $3(3.4)$ & $4(3.4)$ & \\
\hline - Undetermined, n (\%) & $44(56.4)$ & $34(43.5)$ & \\
\hline \% Treg / total lymphocytes at admission & $0.0224 \pm 0.0177$ & $0.0197 \pm 0.0112$ & 0.962 \\
\hline$\%$ Treg / total lymphocytes, $48 \mathrm{~h}$ & $0.0715 \pm 0.0133$ & $0.0231 \pm 0.0173$ & $<0.0001$ \\
\hline$\%$ Treg / total lymphocytes, $72 \mathrm{~h}$ & $0.0709 \pm 0.0064$ & $0.0273 \pm 0.0183$ & $<0.0001$ \\
\hline \% Treg / total lymphocytes, 7 days & $0.0515 \pm 0.0189$ & $0.0209 \pm 0.0172$ & 0.001 \\
\hline
\end{tabular}

(OR: 0.24; 95\% CI: 0.02-0.51; $p<0.0001$ ) were independently associated with infections during hospitalization after adjusting by age, previous history of hypertension, dyslipemia, atrial fibrillation, leukocyte counts, glucose and fibrinogen levels, high-sensitive C-reactive protein levels, basal NIHSS and cardioembolic stroke.

\section{Circulating Treg and serum levels of IL-10}

We found a positive correlation between Treg and IL-10 levels for the 4 time points analyzed. However, this association was stronger the later after stroke during the first 7 days (Fig. 3).

\section{Discussion}

This study evaluated the relationship between circulating levels of Treg (defined as CD4+/CD25+/CD127) and brain injury in IS patients. Circulating Treg levels at 48 and $72 \mathrm{~h}$ were independently associated with good functional outcome at 3 months. This favourable effect on the primary endpoint was supported by positive effects observed on the infarct volume, END and reduction of infections during hospitalization.

The results showed higher levels of IL-10 in patients with ischemic stroke compared with controls. A previous study comparing IL-10 serum levels between stroke patients and healthy population [24] reported contrary results, finding lower levels of IL-10 in stroke patients compared to controls. In this research work, it is noted that small number of patients were included (42 stroke patients and 39 healthy control subjects), in which the mean age of these patients is about 10 years younger than the patients included in our study. In addition, different exclusion criteria were used as active infection-defining temperature or leukocyte levels. Our results suggest that levels of Treg and IL-10 increase during the acute phase, and could exert a pathophysiological role in IS.

Previous studies in animal models of cerebral ischemia showed an increase of Treg infiltration brain tissue at 

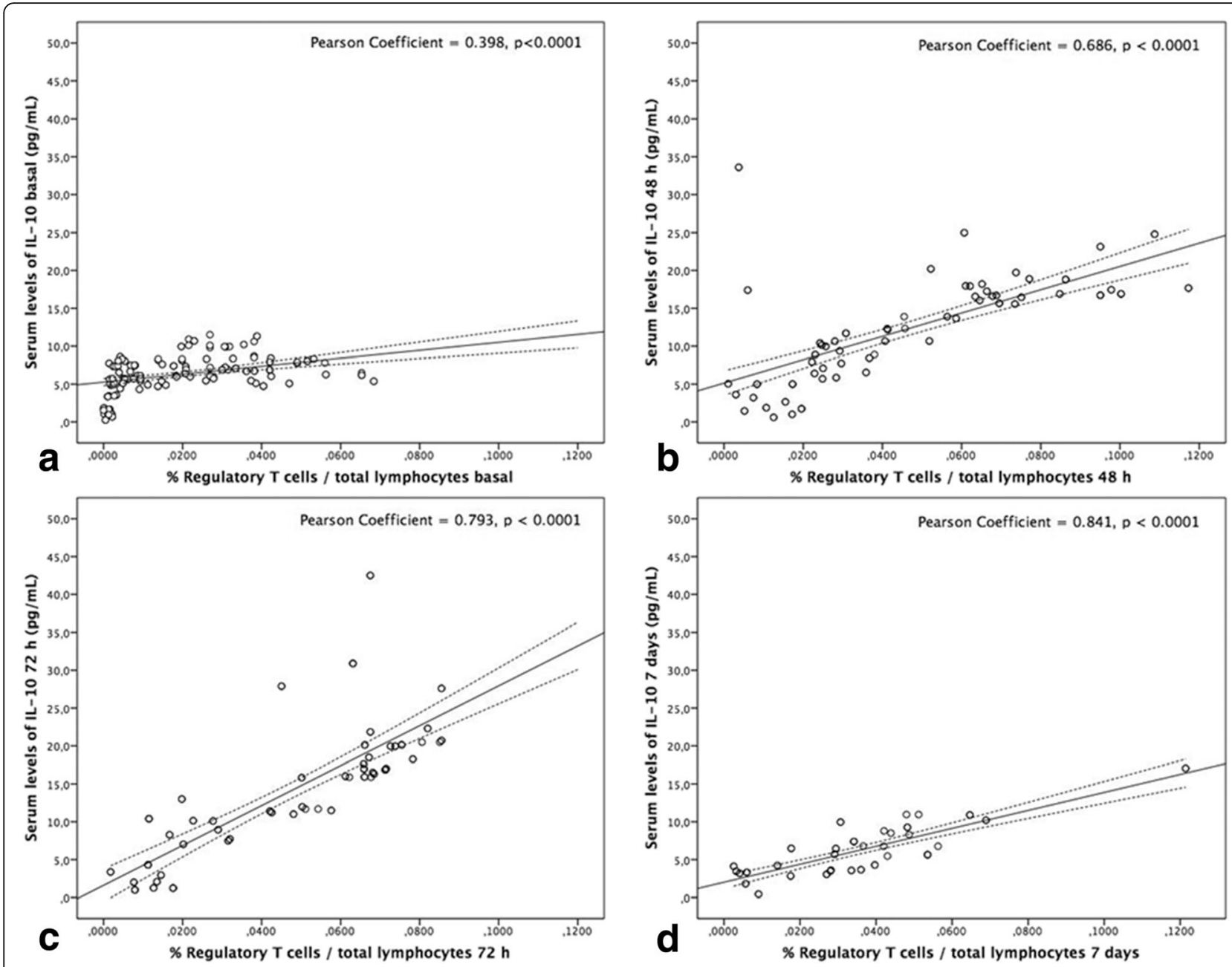

Fig. 3 Association between circulating Treg and serum levels of IL-10 at admission (a), $48 \mathrm{~h}$ (b), $72 \mathrm{~h}$ (c) and day 7 (d)

days 14 and 30 after middle cerebral artery occlusion (MCAO) [25]. In our study we used peripheral blood samples to determinate the temporal pattern of Treg levels during the acute phase of IS. We found that circulating levels of Treg increase during the first 3 days from stroke onset, showing a subsequent but not significant decrease at day 7. Therefore, our results represent a clinical association of the potential role of Treg during the first phase of acute IS. Our results differ from those found in previous small (46 stroke patients and 12 healthy control subjects) and heterogeneous clinical studies (ischemic and hemorrhagic stroke patients were included) that described a decrease in circulating Treg levels at the second day after stroke, followed by a significant increase at day 7 [16].

We found that higher levels of Treg during the acute phase of IS were independently associated with good functional outcome at 3 months. Our results disagree to those reported by Urra et al. [16] that did not find a relationship between Treg levels and functional outcome of
IS patients. The difference could be explained by the small sample size limitation evaluated, the inclusion of ischemic and hemorrhagic stroke patients in that study, and the exclusion criteria used. To the best of our knowledge, this is the first prospective study that specifically analyzed the association between circulating Treg during the acute phase of IS and long-term outcome.

Our results showed that patients with END had lower levels of Treg during the acute phase of stroke. The sample size of patients who suffered END was not enough to perform a multivariate analysis to determinate whether the effect of Treg on END could be a direct cause or if it acts as a surrogate marker. Lower IL-10 levels were associated with clinical worsening [26], but we did not find previous studies analyzing the relationship between END and Treg, so this aspect should be investigated in further studies.

We also studied the association between Treg levels and infarct volume, since it has not been previously reported in literature. We found that higher levels of Treg 
were related with smaller infarct volume. These results also suggest a potential beneficial role of Treg in acute IS, probably by decreasing inflammation which is reflected in a reduction of infarct volume.

Several mechanisms have been proposed for Treg in stroke [27, 28] such as the production of antiinflammatory cytokines, elimination through granzymes and perforins and metabolic mechanisms. In the context of experimental cerebral ischemia, several studies have demonstrated that IL-10 is a key neuroprotective cytokine involved in the regulation of post-stroke neuroinflammation $[9,29]$. In the brain, Treg together with B regulatory (Breg) cells and microglial/monocytes represent the main sources of IL-10. Previous studies in animal models of cerebral ischemia have confirmed the role of IL-10 as a mediator of the protective effect mediated by Treg $[29,30]$. In fact, preclinical strategies directed towards the increase of lymphocyte IL-10 production $[30,31]$ or exogenous IL-10 administration have shown to improve outcome [29]. Therefore, we studied the relationship between Treg and IL-10 levels in IS patients. We found a correlation between IL-10 and Treg levels at admission, 48 and $72 \mathrm{~h}$, and day 7 , supporting the possible anti-inflammatory role of Treg by increasing IL-10 levels, demonstrated in preclinical studies $[29,30]$. Previous clinical studies have established a positive association between higher IL-10 levels during the acute phase and good outcome in IS [26, 32-36]. Nevertheless, no studies had previously investigated the role of IL-10 as a possible mediator of Treg in acute phase of IS in humans.

Other objective of this study was to evaluate the effect of Treg on the risk of systemic infections. Systemic infections are frequent complications during the acute phase of stroke (7-35\%, depending on the series) [37], and its presence worsens the long-term outcome [3743]. Some authors proposed that stroke may induce a systemic immunosuppression that could increase the risk of infections [39]. However, the underlying mechanisms that result in widespread immunosuppression after stroke and subsequent systemic infections are unknown. A study has observed lymphopenia and increased apoptosis of Th lymphocytes, cytotoxic $\mathrm{T}$ lymphocytes and B lymphocytes at early phases of stroke [37]. Increased levels of cortisol and metanephrine have been also related with the risk of infections after stroke $[44,45]$. It has been described that during the first hours after stroke, pro-inflammatory cytokines are upregulated (IL-6, IL-1, TNF $\alpha$, IL-8, MCP-1, etc) [46]. This inflammation stimulates both the hypothalamicpituitary-adrenal axis and sympathetic nervous system, which suppresses immune cell function and can be related to systemic downregulation of the immune system [37]. On the other hand, a manuscript by Hug et al. [47] has already described the impact of stroke volume in a clinical cohort on lymphocyte counts. This study identified infarct volume as a major determiner of the extent of post-ischemic lymphocytopenia and monocyte dysfunction, which are markers of susceptibility to infection.

Treg are a subpopulation of cells with immunosuppressive effects $[48,49]$, so these cells could be related with the risk of infections during the acute phase of stroke. Nevertheless, preclinical studies in animal models [50] demonstrated that exogenous administration of Treg does not exacerbate immunosuppression after cerebral ischemia, and exogenous Treg administration may improve immune status after induction of ischemia. Other clinical studies [16] did not find association between Treg levels with the development of infections after stroke, so this aspect is still unclear. In our study we tried to establish the association between circulating Treg levels and development of infections during hospitalization.

We found that the presence of infections during the acute phase of stroke was associated with poor longterm outcome, as previously reported [37]. Patients with systemic infections showed higher body temperature at 24 and $48 \mathrm{~h}$, suggesting that infections were early developed after ischemic stroke. Interestingly, both lower Treg levels at 48 and $72 \mathrm{~h}$ were independently associated to development of infections after stroke. In fact, most of the infections in our patients were detected during the early phase of stroke (first-second day), when Treg have not achieved their highest levels. In this regard, previous preclinical studies in animal models of cerebral ischemia have demonstrated that Treg exogenous administration improves immune system function, reducing the risk of spontaneous infections after MCAO [32, 49]. Our results confirm this effect described in animal models of cerebral ischemia, suggesting a possible protective role of Treg in the risk of infections, or at least not a deleterious effect.

Finally, our study has some weaknesses: First, we used CD4+, CD25+ and CD127- as membrane markers for Treg. Most authors proposed that FoxP3 is the most specific marker for Treg, but FoxP3 is an intracellular protein, so it cannot be used to isolate human Treg for functional studies or in vivo expansion for cellular therapy. We used the non-expression of CD127, which has previously demonstrated to be directly related with FoxP3 levels [21, 23]. Second, we determined Treg levels in peripheral blood but we have not demonstrated that these cells are infiltrating the brain tissue after IS. For this objective, invasive techniques would be needed to confirm the presence of Treg in ischemic lesion region. Third, Treg levels were only determined during the acute phase of IS. We did not evaluated long-term 
temporal profile of these cells. Finally, we have only evaluated final infarct volume (and not the brain edema), because we did not perform halftime neuroimaging studies unless the patient developed END.

\section{Conclusions}

We found an independent association between Treg levels and good functional outcome at 3 months in IS patients. Treg levels increase after stroke and this increase was closely associated to protective effects; higher levels of these cells were associated to better functional outcome, smaller infarct volume, lower risk of END and infections during hospitalization. Treg were also correlated with IL-10 levels, supporting that this antiinflammatory cytokine may play an important role in the beneficial effects of these cells after ischemic stroke. Therefore, Treg may be considered a potential therapeutic target in acute ischemic stroke. Finally, due to the potential relevance of Treg in stroke and other diseases, new therapeutic strategies able to increase these cells could be developed in the future.

\section{Abbreviations}

CT: Computed tomography; END: Early Neurological deterioration; IS: Ischemic stroke; MCAO: Middle cerebral artery occlusion; MRI: Magnetic Resonance Imaging; mRS: Modified Rankin scale; NIHSS: National institute of Health Stroke Scale; rtPA: Recombinant plasminogen activator;

Treg: Regulatory T cells

\section{Acknowledgements}

Not applicable.

\section{Authors' contributions}

MS-C, JC and TS designed and coordinated the study, participated in the analyzed the data and manuscript preparation. MS-C, ER-C, IL-D, SA-R, MR-Y and MP-M collected the data, and have been involved in the statistical analysis. RI-R, MR-P, PH, MP-L and IL-L have been involved in the statistical analysis, interpretation and processed data. MS-C, JC, PH, TS and FC participated in the data analysis, statistical treatment, and have been involved in revising the manuscript for important intellectual content. All authors have critically read and approved the submitted manuscript.

\section{Funding}

SAF2014-56336-R and SAF2017-84267-R; GRC2014/027 and IN607A2018/3, PIE13/00024; PI17/01103, RETICS-INVICTUS-PLUS (RD16/0019) and European Union FEDER. F. Campos (CP14/00154), T. Sobrino (CPII17/00027), I. LópezLoureiro (IFI15/00111), M. Rodríguez-Pérez (IN606A-2018/031). Sponsors did not participate in study design, collection, analysis, or interpretation of the data.

\section{Availability of data and materials}

The datasets analyzed during the present study will be available from the corresponding author upon reasonable request based on the guidelines of the Ethics Committee of the Servizo Galego de Saúde.

\section{Ethics approval and consent to participate}

This research was carried out in accordance with the Declaration of Helsinki of the World Medical Association (2008), approved by the Ethics Committee of the Servizo Galego de Saúde. Written informed consent was obtained from each patient after full discussion of the procedures. For patients who not being able to provide informed consent, their next of kin was contacted, discussed about study participation, and written informed consent was acquired.
Consent for publication

Not applicable.

\section{Competing interests}

The authors declare that they have no competing interests.

Received: 4 October 2019 Accepted: 19 February 2020

Published online: 28 February 2020

\section{References}

1. Mozaffarian D, Benjamin EJ, Go AS, American Heart Association Statistics Committee and Stroke Statistics Subcommittee, et al. Heart disease and stroke statistics--2015 update: a report from the American Heart Association. Circulation. 2015;131:e29-e322.

2. Rodríguez-Castro E, López-Dequit I, Santamaría-Cadavid M, et al. Trends in stroke outcomes in the last ten years in a European tertiary hospital. BMC Neurol. 2018;18:164

3. Wahlgren N, Ahmed N, Dávalos A, Hacke W, Millán M, Muir K, SITS Investigators, et al. Thrombolysis with alteplase 3-4.5 h after acute ischaemic stroke (SITS-ISTR): an observational study. Lancet. 2008;372:1303-9.

4. Wang $Q$, Tang $X N$, Yenari MA. The inflammatory response in stroke. J Neuroimmunol. 2007;184:53-68.

5. Rosenberg GA. Ischemic brain edema. Prog Cardiovasc Dis. 1999:42:209-16.

6. Siesjo BK, Siesjo P. Mechanisms of secondary brain injury. Eur J Anaesthesiol. 1996:13:247-68.

7. del Zoppo GJ. Acute anti-inflammatory approaches to ischemic stroke. Ann N Y Acad Sci. 2010;1207:143-8.

8. Chen S, Wu H, Klebe D, Hong Y, Zhang J, Tang J. Regulatory T cell in stroke: a new paradigm for immune regulation. Clin Dev Immunol. 2013;2013: 689827.

9. Liesz A, Suri-Payer E, Veltkamp C, Doeer H. Regulatory T cells are key cerebroprotective immunomodulators in acute experimental stroke. Nat Med. 2009;15:192-9.

10. Brea D, Agulla J, Rodríguez-Yáñez M, Barral D, Ramos-Cabrer P, Campos F et al. Regulatory $T$ cells modulate inflammation and reduce infarct volume in experimental brain ischaemia. J Cell Mol Med. 2014;18:1571-9.

11. Hug A, Liesz A, Muerle B, et al. Reduced efficacy of circulating costimulatory cells after focal cerebral ischemia. Stroke. 2011:42:3580-6.

12. Yan J, Greer JM, Etherington GP, Cadigan GP, Cavanagh H, Henderson RD, et al. Immune activation in the peripheral blood of patients with acute ischemic stroke. J Neuroimmunol. 2009;206:112-7.

13. Li Q, Wang Y, Yu F, Wang YM, Zhang C. Hu C. peripheral Th17/Treg imbalance in patients with atherosclerotic cerbral infarction. Int J Clin Exp Pathol. 2013;6:1015-27.

14. Yan J, Read SJ, Henderson R, Hull R, O'Sullivan J, McCombe P, Greer JM. Frecuency and function of regulatory $T$ cells after ischemic stroke in humans. J Neuroimmunol. 2012:243:89-94.

15. Wrigen M, Björkbacka $H$, Andersson L, Ljungcrantz I, Fredrikson GN, Persson $M$, et al. Low levels of circulating CD4+FoxP3+ T cells are associated with an increased risk for development of myocardial infarction but not for stroke. Arterioscler Thromb Vasc Biol. 2012:32:2000-4.

16. Urra X, Cervera A, Villamallor M, Planas AM, Chamorro A. Harms and benefits of lymphocyte subpopulations in patients with acute stroke. Neuroscience. 2009:158:1174-83.

17. Workman CJ, Szymczak-Workman AL, Collison LW, Pillai MR, Vignali DA. The development and function of regulatory T cells. Cell Mol Life Sci. 2009;66: 2603-22.

18. Alonso de Leciñana M, Egido JA, Casado I, Ribó M, Dávalos A, Masjuan J. et al. Guidelines for the treatment of acute ischaemic stroke. Neurologia. 2014:29:102-22.

19. Adams HP Jr, Bendixen BH, Kappelle J, Biller J, Love BB, Gordon L, et al. Classification of subtype of acute ischemic stroke. Definitions for use in a multicenter clinical trial. TOAST. Trial of org 10172 in acute stroke treatment. Stroke. 1993;24:35-41.

20. Pedraza S, Puig J, Blasco G, Daunis-Estadella J, Boada I, Bardera A, et al. Reliability of the $A B C / 2$ method in determining acute infarct volume. J Neuroimaging. 2012;22:155-9.

21. Liu W, Putman AL, Xu Yu Z, Szot GL, Lee MR, Zhu S, et al. CD127 expression inversely correlates with FoxP3 and suppressive function of human CD4+ Treg cells. J Exp Med. 2006;20:1701-11. 
22. Peters JH, Preijers FW, Woostenenik R, Hilbrands LB, Koenen HJ, Joosten I. Clinical grade Treg: GMP isolation, improvement of purity by CD127 depletion, Treg expansion, and Treg cryopreservation. PLoS One. 2008;3: e3161.

23. Seddiki N, Santner-Nonan B, Martninson J, Zaunders J. Expression of interleukin (IL)-2 and IL-7 receptors discriminates between human regulatory and activated T cells. J Exp Med. 2006;203:1693-700.

24. Perini F, Marro M, Alecci M, Galloni E, Marchi M, Tosjo V. Temporal profile of serum anti-inflammatory and pro-inflammatory interleukins in acute ischemic stroke. Neurol Sci. 2001:22:289-96.

25. Stubbe T, Ebner F, Ritcher D, et al. Regulatory T-cells accumulate and proliferate in the ischemic hemisphere for up to 30 days after MCAO. J Cereb Blood Flow Metab. 2013;33:37-47.

26. Vila N, Castillo J, Dávalos A, Esteve A, Planas AM, Chamorro A. Levels of antiinflammatory cytokines and neurological worsening in acute ischemic stroke. Stroke. 2003;34:671-5.

27. Otsubo K, Kanegane H, Kamachi $Y$, et al. Identification of FOXP3-negative regulatory T-like (CD4(+)CD25(+)CD127(low)) cells in patients with immune dysregulation, polyendocrinopathy, enteropathy, X-linked syndrome. Clin Immunol. 2011;141:111-20.

28. Liesz A, Hu X, Kleinschnitz C, Offner H. Functional role of regulatory lymphocytes in stroke. Facts and controversies. Stroke. 2015;46:1422-30.

29. Spera PA, Ellison JA, Fenersten GZ, Barone FC. IL-10 reduces rat brain injury following focal stroke. Neurosci Lett. 1998;251:189-92.

30. Liesz A, Zhou W, Na S, Hammerling GJ. Boosting regulatory T cells limits neuroinflammation in permanent cortical stroke. J Neurosci. 2013;33:17350-62.

31. Na SY, Marckso E, Liesz A, Hunig T, Velktamp R. Amplification of regulatory T-cells using a CD28 superagonist reduces brain damage after ischemic stroke in mice. Stroke. 2015:46:212-20.

32. Li P, Gan Y, Sun BL, Zhang F, Lu B, Gao Y, et al. Adoptive regulatory T-cell therapy protects against cerebral ischemia. Ann Neurol. 2013;74:458-71.

33. Protti GG, Gagliardi RJ, Forte WC, Spravieri SR. IL-10 may protect against progressing injury during acute phase of ischemic stroke. Arq Neuropsiquiatr. 2013;71:846-51

34. Singh HV, Pandey A, Shrivastova AK, Raizade A, Singh SK, Singh N. Prognostic value of neuron specific enolase and IL-10 in ischemic stroke and its correlation with degree of neurological deficit. Clin Chim Acta. 2013; 419:136-8.

35. Chang LT, Yuen C, Lian CW, Lu CH, Chang WN, Youssef AA, et al. Link between IL-10 level and outcome after ischemic stroke. Neuroimmunomodulation. 2010;17:223-8.

36. Rodríguez-Yáñez M, Castellanos M, Sobrino T, Brea D, Ramos-Cabrer P, Pedraza S, et al. Interleukin-10 facilitates the selection of patients for systemic thrombolysis. BMC Neurol. 2013;13:62.

37. Chamorro A, Urra X, Planas AM. Infection after acute ischemic stroke: a manifestation of brain-induced immunodepression. Stroke. 2007;38:1097-103.

38. Davenport RJ, Dennis MS, Weelwood L, Warlow CP. Complications after acute stroke. Stroke. 1996;27:415-20.

39. Grau AJ, Buggle F, Schitrler P, Spiel M, Lichy C, Hacke W. Fever and infection early after ischemic stroke. J Neurol Sci. 1999;171:115-20.

40. Langhorne P, Stott DJ, Robertson L, McDonald M, Jones L, McAlpine C, et al. Medical complications after stroke: a multicenter study. Stroke. 2000;31: 1223-9.

41. Katzan IL, Cebul RD, Husak SH, Dawson NV, Baker DW. The effect of pneumonia on mortality among patients hospitalized for acute stroke. Neurology. 2003;60:620-5.

42. Heuschmann PU, Kolominsky-Rabas PL, Misselwitz B, Hermanek P, Leffmann C, Jauzen RW, et al. Predictors of in-hospital mortality and attributable risks of death after ischemic stroke: the German stroke registers study group. Arch Intern Med. 2004;164:1761-8.

43. Vargas M, Horcajada J, Obach V, Revilla M, Cervera A, Torres F, et al. Clinical consequences of infection in patients with acute stroke: is it prime time for further antibiotic trials? Stroke. 2006;37:461-5.

44. Wilckens T, De Rijk R. Glucocorticoids and immune function: unknown dimensions and new frontiers. Immunol Today. 1997;18:418-24.

45. Elenkov IJ, Wilder RL, Chrousos GD, Vizi ES. The sympathetic nerve an integrative interface between two supersystems: the brain and the immune system. Pharmacol Rev. 2000;52:595-638.

46. Rodríguez-Yáñez M, Castillo J. Role of inflammatory markers in brain ischemia. Curr Opin Neurol. 2008;21:353-7.
47. Hug A, Dalpke A, Wieczorek N, Giese T, Lorenz A, Auffarth G, Liesz A, Veltkamp R. Infarct volume is a major determiner of post-stroke immune cell function and susceptibility to infection. Stroke. 2009;40:3226-32.

48. O'Garra A, Vieira P. Regulatory T cells and mechanisms of immune system control. Nat Med. 2004;10:801-5.

49. Emsley $\mathrm{H}$, Hopkins SJ. Post-stroke immunodepression and infection: an emerging concept. Infect Disord Drug Targets. 2010;10:91-7.

50. Li P, Mao L, Zhou G, Leak RK, Sun B, Chen J, et al. Adoptive regulatory T cell therapy preserves systemic inmmune homeostasis after cerebral ischemia. Stroke. 2013;44:3509-15.

\section{Publisher's Note}

Springer Nature remains neutral with regard to jurisdictional claims in published maps and institutional affiliations.
Ready to submit your research? Choose BMC and benefit from:

- fast, convenient online submission

- thorough peer review by experienced researchers in your field

- rapid publication on acceptance

- support for research data, including large and complex data types

- gold Open Access which fosters wider collaboration and increased citations

- maximum visibility for your research: over $100 \mathrm{M}$ website views per year

At BMC, research is always in progress.

Learn more biomedcentral.com/submissions 